\section{Generation of High- Quantity and Quality Tag/Ditag cDNAs for SAGE Analysis}

\author{
BioTechniques 31:348-354 (August 2001)
}

\begin{abstract}
The serial analysis of gene expression (SAGE) technique is an important tool for genome-wide gene expression analysis. However, the requirement of a large amount of $m R N A$ for the analysis and the difficulties in generating high-quality tag and ditag fragments for the construction of a SAGE library often interfere with the successful performance of the SAGE technique. We developed two procedures to solve these issues: (i) introducing low-cycle PCR amplification of the 3' cDNA before the $\mathrm{Bs} \mathrm{mFI}$ digestion of the 3' cDNAs and (ii) gel purifying the $\mathrm{BsmFI-released} \mathrm{tag} \mathrm{fragments} \mathrm{be-}$ fore ditag formation. These modifications provide a large quantity of initial $3^{\prime}$ cDNAs and high-quality tags and ditags for the construction of SAGE libraries.
\end{abstract}

\section{INTRODUCTION}

The development of the serial analysis of gene expression (SAGE) technique provides a powerful tool for genome-wide gene analysis (10). Applying the SAGE technique can provide both the qualitative information for the identification of expressed genes in a particular cell population and quantitative information for the identification of differentially expressed genes between different cells. The data generated through the SAGE technique have provided in-depth information to understand the pattern of gene expression at the genome level $(9,11,14)$.

The SAGE technique is essentially designed based on routine molecular biology techniques. However, several steps in the SAGE procedure are very critical for its successful performance. Others have attempted to improve the standard SAGE protocol (1,3,4,6-8, 13). We focused on two issues to im prove the performance of the SAGE: (i) using a low amount of mRNA for SAGE analysis. The standard SAGE protocol requires $5 \mu \mathrm{g}$ mRNA as the starting material for the analysis. In many situations, it is impossible to obtain this amount of mRNA. By applying a low-cycle PCR to amplify the $3^{\prime}$ cDNA, we developed a procedure to perform SAGE analysis with a small quantity of mRNA. (ii) Providing highquality tag/ditags for the construction of a SAGE library. Even with a sufficient amount of the initial materials, the successful construction of the SAGE library is largely affected by the efficient isolation of tag/ditag fragments $(4,7)$. Frequently, the low quality of tag/ditag fragments prevents a successful performance of SAGE analysis. We applied gel purification for the 51-bp DNA fragments containing tags before the ditag formation. As shown by our data, these procedures provide a significant improvement over the standard SAGE protocol. Here, we report the details of these two procedures.

\section{MATERIALS AND METHODS}

\section{Incorporation of PCR to Amplify the $3^{\prime}$ cDNA}

The procedures from RNA extraction to $3^{\prime}$ cDNA isolation were performed essentially following the standard SAGE protocol. In brief, the total RNA was isolated from human bone marrow cells with TRIzOL $^{\circledR}$ reagent (Life Technologies, Rockville, MD, USA). The mRNA was isolated with oligo-dT25 magnetic beads (Dynal, Lake Success, NY, USA). The doublestranded cDNA was synthesized with a cDNA synthesis kit containing Moloney murine leukemia virus (MMLV) reverse transcriptase (Life Technologies). A set of biotin-labeled anchored oligo(dT) primers $\left(5^{\prime}\right.$-ATCTAGAGCG GCCGCdT16G-3', 5'-ATCTAGAGCG GCCGCdT16 A-3', 5'-ATCTAGAGCGGCCGCdT16CA-3'， 5'-ATCTAGAGCGGCCGCdT16CC-3', and 5'-ATCTAGAGCGGCCGCdT16CG-3') was used for reverse transcription (12). The principle of the reaction is that only the anchored primers annealed to the $5^{\prime}$ end of the mRNA poly(A) tail and the anchored nucleotide paired correctly to the nucleotide next to the $\operatorname{poly}(\mathrm{A})$ tail can be extended by reverse transcriptase. Primers that were annealed to the other position along the poly(A) sequence could not be extended because the unpaired anchors block the extension. These features should provide cDNA with long poly(dT) sequences. The double-stranded cDNAs were digested with NlaIII. The $3^{\prime}$ cDNAs were then isolated with streptavidin beads (Dynal). The beads containing the $3^{\prime}$ cDNAs were divided into two tubes and ligated with the standard SAGE linker $1 \mathrm{~A}$ or $1 \mathrm{~B}$. The free linkers remaining after ligation were washed away. The 3' cDNAs were then PCR amplified using Taq DNA polymerase (Takara, Shiga, Japan), with the sense primer of SAGE primer 1 ( $5^{\prime}-\mathrm{GG}$ ATTTGCTGGTGCAGTACA-3') or SAGE primer 2 (5'-CTGCTCGAATTCAAGCTTCT-3') and the antisense primer (5'-ACTATCTAGAGCGGCCGCTT-3') located in the $3^{\prime}$ end of cDNAs incorporated from the anchored oligo(dT) primers.

\section{Gel Purification of Tag Fragments}

Five micrograms of PCR-amplified $3^{\prime}$ cDNAs were digested by $B s m \mathrm{FI}$ and blunt-ended by $P f u$ DNA polymerase (Stratagene, La Jolla, CA, USA) for $1 \mathrm{~h}$ at $65^{\circ} \mathrm{C}$ in a $50-\mu \mathrm{L}$ reaction containing $5 \mu \mathrm{L} 10 \times$ cloned $P f u$ DNA polymerase buffer, $4 \mu \mathrm{L} B s m \mathrm{FI}(2 \mathrm{U} / \mu \mathrm{L}), 1 \mu \mathrm{L} 10$ mM dNTP, $1.5 \mu \mathrm{L}$ cloned $P f u$ DNA polymerase $(2.5 \mathrm{U} / \mu \mathrm{L}), 30 \mu \mathrm{L}$ cDNA, and $8.5 \mu \mathrm{L}$ water. The digested and blunt-ended cDNA sample was extracted with $50 \mu \mathrm{L}$ phenol/chloroform, precipitated with $17 \mu \mathrm{L} 10 \mathrm{M} \mathrm{NH}_{4} \mathrm{OAc}, 2$ $\mu \mathrm{L}$ glycogen, and $184 \mu \mathrm{L} 100 \%$ ethanol. The purified DNA was loaded into a $15 \%$ polyacrylamide gel (Figure 1 ). The 51-bp fragments were sliced from the gel and smashed by centrifugal force. The 51-bp fragments were eluted and precipitated with ethanol.

\section{Formation of Ditags}

The ligation of purified 51-bp fragments to form $102 \mathrm{bp}$ was as follows: $12 \mu \mathrm{L}$ 51-bp fragments with SAGE linker A, $12 \mu \mathrm{L}$ 51-bp with linker B, $1.5 \mu \mathrm{L} 10 \times$ ligase buffer (Invitrogen, Carlsbad, CA, USA), 1.5 $\mu \mathrm{L}$ T4 DNA 


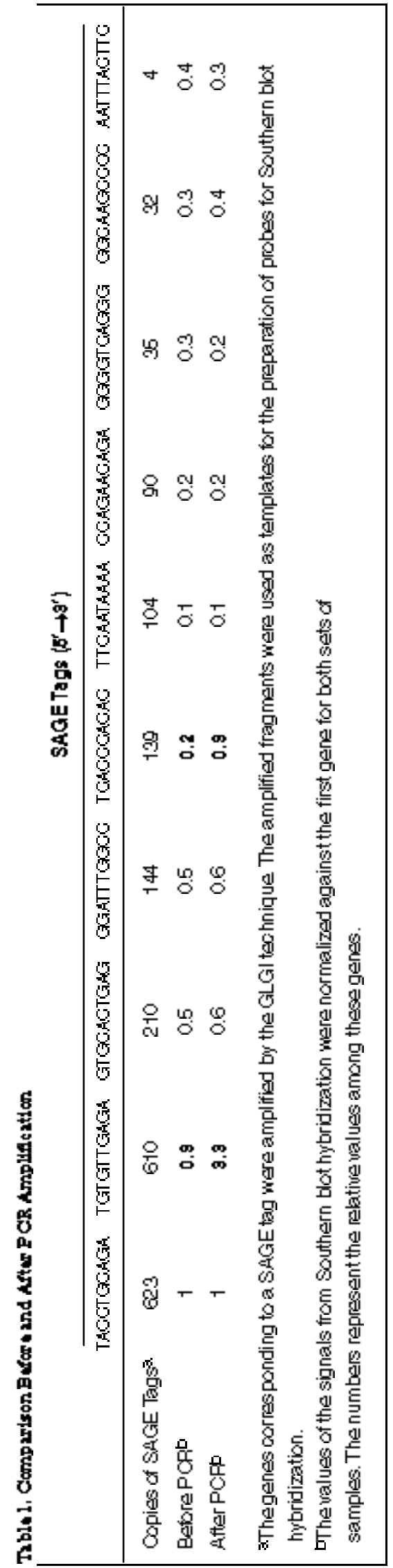

ligase (4 U/ $\mu \mathrm{L}$; Invitrogen). The reaction was incubated for $3 \mathrm{~h}$ at $16^{\circ} \mathrm{C}$. The ligation mixture was purified through phenol/chloroform extraction and ethanol precipitation.

\section{Isolation of Ditags, Concatemeriza- tion of Ditags, Cloning, and Sequencing}

These steps were performed follow ing the standard SAGE protocol, except ditags were isolated from $15 \%$ polyacrylamide gel rather than $12 \%$ to give better separation of 26-bp ditags from the 42-bp linkers. The concatemers were loaded on an $8 \%$ polyacrylamide gel, and fragments of more than $600 \mathrm{bp}$ were isolated and cloned into the pZero vector (Invitrogen). Universal M13 forward and reverse primers were used for PCR. The PCR products were purified and used for sequencing reaction with universal M13 forward primer.

\section{RESULTS AND DISCUSSION}

The cDNAs digested by NlaIII have similar sizes to the majority centered at 256 bp (12). All the $3^{\prime}$ cDNA templates have the same linker sequence at their $5^{\prime}$ end and the same sequences ( $5^{\prime}$ ATCTAGAGCGGCCGC-3') derived from the reverse transcription primer at their $3^{\prime}$ end. Therefore, because the reactions were limited to a low cycle number, the amplified products should generally maintain the quantitative pattern present in the templates in the original $3^{\prime}$ cDNAs. A cDNA sample before and after amplification through $16 \mathrm{cy}-$ cles was used for Southern analysis to show whether this was the case. A set of genes expressed at different abundance identified by SAGE was chosen as the test genes. The $3^{\prime}$ cDNA tem plates corresponding to the SAGE tags were amplified as a single fragment from each reaction by the generation of longer 3' cDNA fragments from SAGE tags for gene identification (GLGI) technique. The amplified fragments were verified by cloning and sequencing and used as templates for the preparation of the probes by random priming (2). The signals in the original sample and in the amplified samples were normalized to the first genes (amplified by GLGI from 5'-TACCTGCAGA-3', 623 tags). The numbers after the normalization reflect the quantitative relationship among these genes in each sample. As shown in Table 1 , among the 10 genes chosen for the comparison, 8 genes maintained the quantitative pattern between the original cDNA and the PCRamplified cDNA. Two genes (tags with 610 copies and tag with 139 copies) show 3- and 4-fold increases after PCR

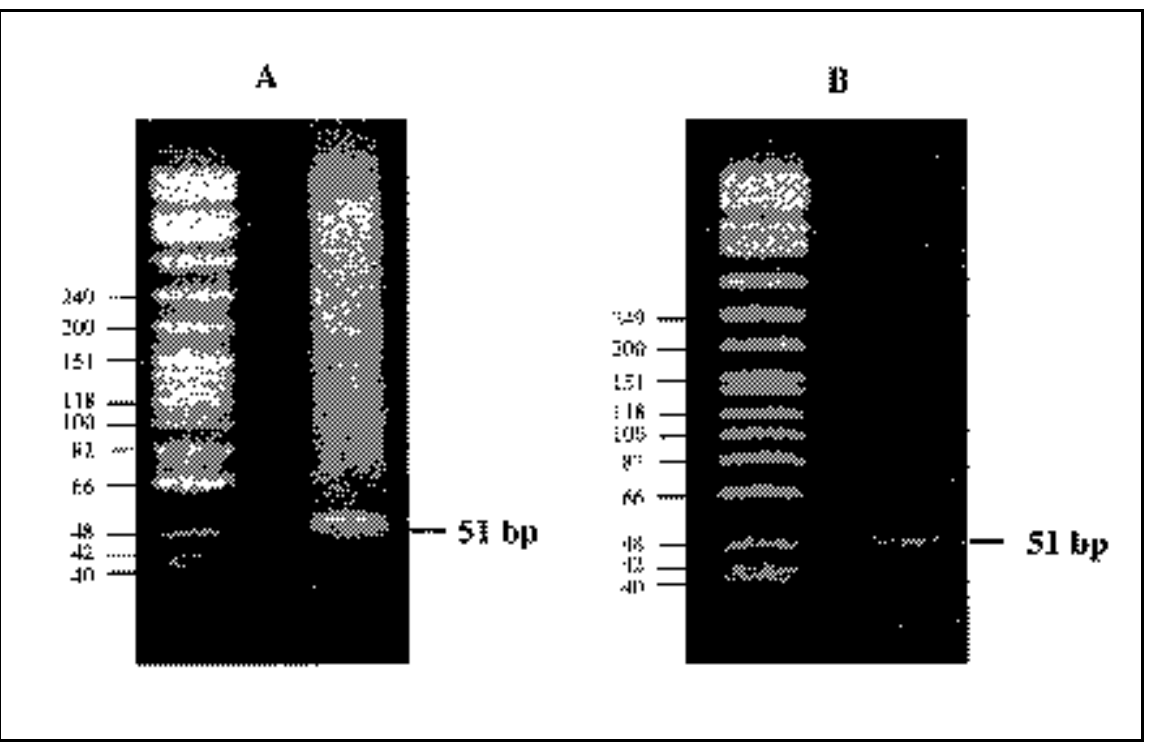

Figure 1. Release of tag fragments from $3^{\prime}$ cDNAs. (A) The 51-bp fragments containing SAGE tags were released from $3^{\prime} \mathrm{cDNAs}$ by $B s m \mathrm{FI}$ digestion. (B) The 51-bp fragments were purified from $10 \%$ polyacrylamide gel before being used for the formation of 102-bp fragments. 


\section{Short Technical Reports}

amplification. These two genes were expressed at a highly abundant level, which might contribute to the biased amplification. These results indicate that under following conditions-(i) using the $3^{\prime}$ cDNA as the templates, (ii) using the same sense and antisense primers for PCR, and (iii) using a lowcycle PCR for amplification-the resulting cDNA will basically maintain the original quantitative pattern. How ever, caution should be taken so that the PCR cycles are kept to a minimum. The ditag fragments were amplified through PCR by Platinum Taq DNA polymerase (Life Technologies), with biotin-labeled SAGE primer $1\left(5^{\prime}-\mathrm{GG}\right.$ ATTTGCTGGTGCAGTACA-3') and SAGE primer $2\left(5^{\prime}\right.$-CTGCTCGAATTCAAGCTTCT-3'). As shown in Figure 2 , the 102-bp fragments were efficiently amplified at fewer than 20 PCR cycles. An important feature of this amplification is the pattern of PCR products: the 102-bp fragments were the dominantly amplified fragments, and there were no fragments smaller than $102 \mathrm{bp}$. In the standard protocols, the PCR amplification of the 102-bp fragments usually generates several strong fragments smaller than the 102 bp. Those smaller fragments may originate from the di-linkers formed in the ligation of linkers to the $3^{\prime}$ cDNAs. These smaller fragments make the gel purification of the 102-bp fragments rather difficult and can contaminate the purified 102-bp fragments. Those contaminated fragments can have potentially inhibitory effects for the downstream steps of concatemerization.

The NlaIII enzyme is rather sensitive to different conditions. It has been observed that NlaIII often has problems in digesting DNA templates because of its short half-life, the temperature for its storage, or its sensitivity to the conditions of digestion. We observed that this enzyme is unable to digest efficiently the gel-purified DNA but can digest efficiently the DNA without gel purification. On the other hand, the NlaIII-digested DNA can be efficiently

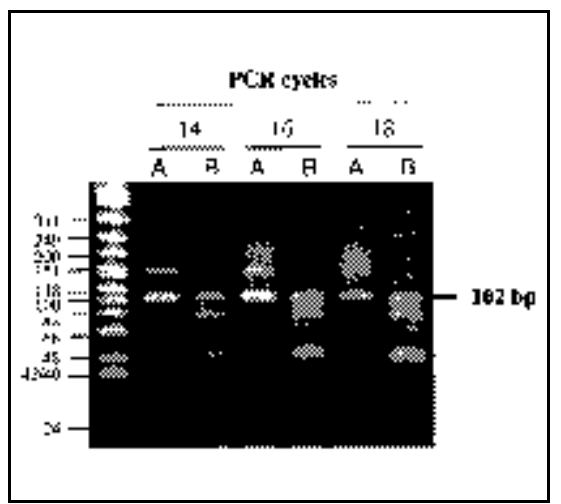

Figure 2. PCR amplification of 102-bp fragments. (A) PCR amplification of 102-bp fragments from the gel-purified and ligated 51-bp fragments. (B) PCR amplification of 102-bp fragments from unpurified and ligated 51-bp fragments. 


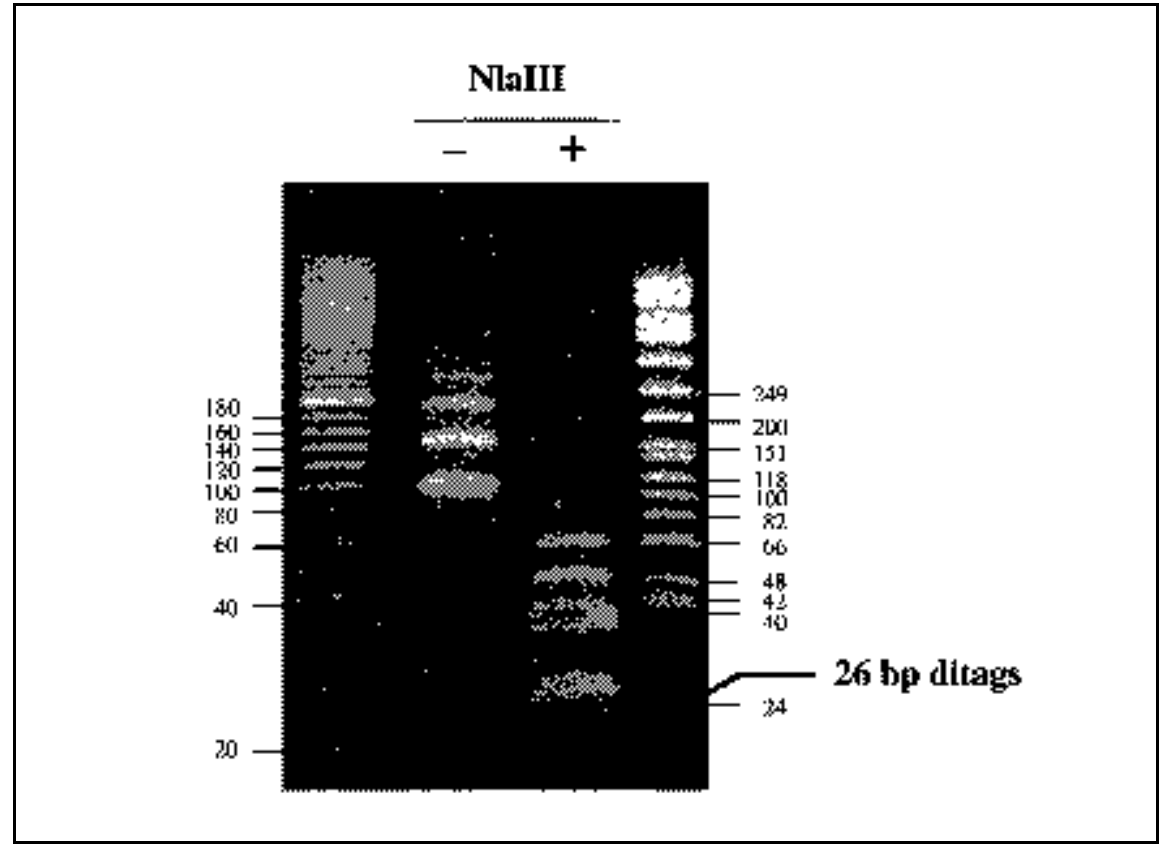

Figure 3. Recovery of the 26-bp ditags. The 26-bp ditags were released from the PCR-amplified 102-bp fragments through NlaIII digestion and recovered through $15 \%$ polyacrylamide gel. re-ligated, regardless of whether or not the NlaIII-digested DNA was gel purified. Based on these observations and the presence of primarily 102-bp fragments in the total PCR products, we decided not to gel purify the 102-bp fragment but rather to digest the 102-bp fragments directly with NlaIII to release the 26 ditags (Figure 3). The 26$\mathrm{bp}$ fragments containing the ditags were gel purified and used for the concatemerization, which generated fragments far longer than $500 \mathrm{bp}$ (Figure 4A). The fragments larger than $600 \mathrm{bp}$ were purified and cloned into pZero vector. More than $80 \%$ of the clones contained insert higher than 500 bp (Figure 4B). The tags from linkers were essentially elim inated from the final SAGE tag data sets (e.g., only two tag copies of linker $\mathrm{A}$ and 16 copies of linker B were detected in 67000 tags collected from one SAGE library generated by myeloid cells. In comparison, tags from linker 


\section{Short Technical Reports}

A.

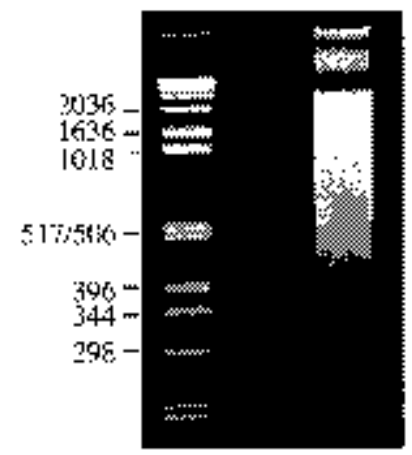

B.

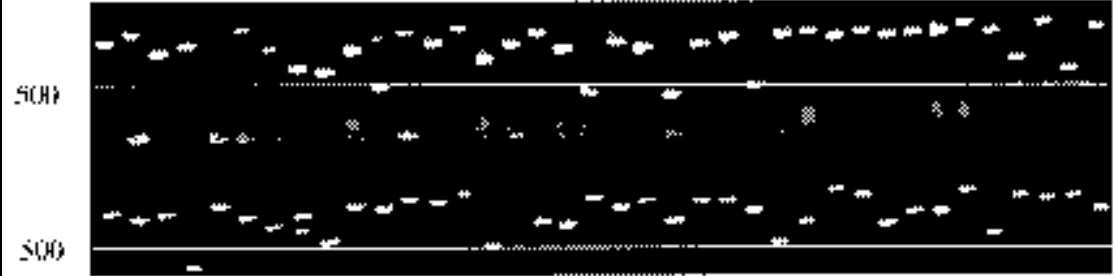

Figure 4. Size distribution of concatemers and cloned inerts. (A) Concatemerization of ditags. The ligated ditag product was loaded into an $8 \%$ polyacrylamide gel. The fragments larger than $600 \mathrm{bp}$ were purified and cloned into pZero vector. (B) PCR amplification of cloned inserts. The majority of clones contained insert size of more than $500 \mathrm{bp}$.

contamination comprise $4 \%-5 \%$ of total SAGE tags collected with the standard SAGE procedures $(5,9)$.

The procedures we developed through this study overcome the limitation of a small amount of initial mRNA for SAGE analysis and significantly improve the quality of tag and ditag fragments for the construction of a high-quality SAGE library. These im provements should result in better performance of SAGE technique for gene analysis. Using this modification, we are routinely performing SAGE analysis starting with $5 \mu \mathrm{g}$ total RNA. Data from sequencing thousands of clones from four different SAGE libraries generated between 70000 and 100000 tags from each library with this procedure.

\section{REFERENCES}

1.Angelastro, J.M., L.P. Klimaschewski, and O.V. Vitolo. 2000. Improved NlaIII digestion of PAGE-purified $102 \mathrm{bp}$ ditags by addition of a single purification step in both the SAGE and microSAGE protocols. Nucleic Acids
Res. 28:e62.

2.Chen, J-J., J.D. Rowley, and S.M.Wang. 2000. Generation of longer cDNA fragments from serial analysis of gene expression tags for gene identification. Proc. Natl. Acad. Sci. USA 97:349-353.

3.Datson, N.A., J. van der Perk-de Jong, M.P. van den Berg, E.R. de Kloet, and E. Vreugdenhil. 1999. MicroSAGE: a modified procedure for serial analysis of gene expression in limited amounts of tissue. Nucleic Acids Res. 27:1300-1307.

4.Kenzelmann, M. and K. Muhlemann. 1999. Substantially enhanced cloning efficiency of SAGE (serial analysis of gene expression) by adding a heating step to the original protocol. Nucleic Acids Res. 27:917-918.

5.Lal, A., A.E. Lash, S.F. Altschul, V. Velculescu, L. Zhang, R.E. McLendon, M.A. Marra, C. Prange et al. 1999. A public database for gene expression in human cancers. Cancer Res. 59:5403-5407.

6.Peters, D.G., A.B. Kassam, H Yonas, E.H. O'Hare, R.E. Ferrell, and A.M. Brufsky. 1999. Comprehensive transcript analysis in small quantities of mRNA by SAGE-lite. $\mathrm{Nu}-$ cleic Acids Res. 27:e39.

7.Powell, J. 1998. Enhanced concatemer cloning-a modification to the SAGE (serial analysis of gene expression) technique. Nucleic Acids Res. 26:3445-3446.

8.van den Berg, A., J. van der Leij, and S. Poppema. 1999. Serial analysis of gene ex- pression: rapid RT-PCR analysis of unknown SAGE tags. Nucleic Acids Res. 27:e17.

9.Velculescu, V.E., S.L. Madden, L. Zhang, A.E. Lash, J. Yu, C. Rago, A. Lal, C.J. Wang et al. 1999. Analysis of human transcriptomes. Nat. Genet. 23:387-388.

10.Velculescu, V.E., L. Zhang, B. Vogelstein, and K.W. Kinzler. 1995. Serial analysis of gene expression. Science 270:484-487.

11. Velculescu, V.E., L. Zhang, W. Zhou, J. Vogelstein, M.A. Basrai, D.E. Bassett, P. Hieter, B. Vogelstein, and K.W. Kinzler. 1997. Characterization of the yeast transcriptome. Cell 88:243-251.

12.Wang, S.M., S.C. Fears, L. Zhang, J.-J. Chen, and J.D. Rowley. 2000. Screening poly(dA/dT) cDNAs for gene identification. Proc. Natl. Acad. Sci. USA 97:4162-4167.

13. Wang, S.M. and J.D. Rowley. 1998. A strategy for genome-wide gene analysis: integrated procedure for gene identification. Proc. Natl. Acad. Sci. USA 95:11909-11914.

14.Zhang, L., W. Zhou, V.E. Velculescu, S.E. Kern, R.H. Hruban, S.R. Hamilton, B. Vogelstein, and K.W. Kinzler. 1997. Gene expression profiles in normal and cancer cells. Science 276:1268-1272.

S.L. and J.C. contributed equally to this paper. We thank Dr. Janet D. Rowley for her encouragement. This research was supported in part by National Institutes of Health grant no. CA78862-01 (J.D.R. and S.M.W.), American Cancer Society grant no. IRG41-40 (S.M.W.), and the G. Harold and Lelia Y. Mathers Charitable Foundation (S.M.W.). Address correspondence to Dr. San Ming Wang, Section of Hematology/ Oncology, University of Chicago Medical Center, 5841 S. Maryland MC2115, Chicago, IL 60637, USA. e-mail: swang1@ midway.uchicago.edu

Received 10 October 2000; accepted 16 February 2001.

\section{Sanggyu Lee, Jianjun Chen, Guolin Zhou, and San Ming Wang \\ University of Chicago \\ Medical Center \\ Chicago, IL, USA}

